\title{
Evaluation of a Acute Febrile Illness Surveillance Network (GAFINet), South Korea.
}

\author{
Heeyoung Lee ${ }^{1}$, Yeon-Hee Sung ${ }^{2}$, Kyoung-Ho Song ${ }^{1}$, Yang Lee $\mathrm{Kim}^{3}$, Jeong Yeon $\mathrm{Kim}^{4}$, Jieun \\ $\mathrm{Kim}^{5}$, Won Suk Choi 6 \\ ${ }^{1}$ Center for preventive medicine and public health, Seoul National Bundang Hospital, Seongnam, Korea (the Republic of), ${ }^{2}$ GIDCC, Seongnam, Korea \\ (the Republic of), ${ }^{3}$ The Catholic Univ. of Korea Uijeongbu Mary's hospital, Uijeongbu-si, Korea (the Republic of), ${ }^{4} 4 \mathrm{GPMC}$ (Gyeonggi Provincial \\ Medical Center, Suwon, Korea (the Republic of), ${ }^{5}$ Hanyang University College of Medicine, Seoul, Korea (the Republic of), ${ }^{6}$ Korea University College \\ of Medicine, Ansan, Korea (the Republic of)
}

\section{Objective}

The purpose of this study is to describe and evaluate the results of the GAFINET(Gyeonggi Acute Febrile Illness Surveillance Network) operated for one year.

\section{Introduction}

After MERS outbreak in 2015, the provincial government and infectious disease control center (GIDCC) initiated an emergency department (ED) based Gyeonggi-do provincial acute febrile illness (AFI) surveillance network (GAFINet) to monitor for a subsequent outbreak of emerging or imported infectious diseases since September 2016. Following pilot operation from September to December 2016, the operation was run for one year from June 2017 to May 2018.

GAFINet Initiative involves ten hospitals, consisted of four university-affiliated hospitals and six provincial medical centers in Gyeonggi-do province. These hospitals participated in this network voluntarily.

\section{Methods}

Periodic surveillance for finding AFI patients in ED of participating hospitals was performed prospectively (Figure 2). AFI was defined as 1) fever: body temperature $\geq 38{ }^{\circ} \mathrm{C}$ at admission, or 2) chief complaint of febrile or chilling sensation. Demography of patients and chief complaints were investigated in this first step. Cases were classified into six categories based on their clinical diagnoses: 1) respiratory AFI [AFRI], 2) gastroenteric AFI [AFGI], 3) exanthematic AFI [AFEI], 4) other infectious AFI, 5) noninfectious AFI, and 6) unclassified AFI. Participating infectious diseases specialists regularly reviewed and reformed this classification via web based system.

Nosocomial AFI cases or the patients transferred from another hospital were excluded. When a patient had a history of international travel or he/she were undiagnosed in three days after ED admission, more comprehensive information including history and final diagnosis were obtained. For a baseline data, age- and sex-stratified ED visits were also gathered weekly. The proportion of AFI cases per 1000 visits was determined for one week period and analyzed by febrile diseases categories with age-stratification. Characteristics of cases with international travel histories or undiagnosed cases were also described separately.

The results were presented to participating researchers as visualized dashboards in Web-based systems.

Also we compared the trend and peak time of GAFINET data with the surveillance data of KCDC for influenza, hand foot and mouth disease, and Tsutsugamushi disease.

\section{Results}

The total number of patients in the emergency room in 10 hospitals was 366,695 for one year. Among them, 40,897 patients were diagnosed with acute febrile illness (11.2\%). 47.8\% were under 10 years of age, and 508 - 1,769 patients were fever - related patients per weak. The number of patients with foreign travel was $0-11$ in each week, and the number of patients with unknown fever in the week was $0-6$.

1 to 9 years of age accounted for the largest proportion $(27.6 \%-48.3 \%)$ of acute febrile patients. The most common infectious diseases were acute febrile respiratory disease, and other acute febrile infections and acute febrile gastrointestinal disease are

ISDS Annual Conference Proceedings 2019. This is an Open Access article distributed under the terms of the Creative Commons AttributionNoncommercial 4.0 Unported License (http://creativecommons.org/licenses/by-nc/3.0/), permitting all non-commercial use, distribution, and reproduction in any medium, provided the original work is properly cited. 
ISDS 2019 Conference Abstracts

followed. In acute febrile respiratory disease and acute febrile illness, seasonal pattern was seen. A total of 157 patients with acute febrile illnesses with overseas travel ability, 0 - 11 persons per week, consistently occurred. Acute gastroenteritis (AGE) was the most frequent diagnosis in 28 patients (17.8\%), fever of unkonwn origin(FUO) was 25(15.9\%), Acute pharyngotonsilitis 17(10.8\%), viral infection $17(10.8 \%)$, Influenza $7(4.5 \%)$ and colitis $5(3.2 \%)$. The cause of fever was not found in 30 of the acute febrile patients with overseas travel ability. A total of 77 patients with acute febrile disease were admitted to the hospital.

In comparison with national surveillance data, the trend of occurrence of influenza in GAFINet and national data was similar. Both data peaked in the first week of 2018. Also trend of hand-foot and mouth disease(HFMD) was similar in two data. But peak weak was little different in both data(CDC at 30 weeks, GAFINett at 29 weeks in 2017). In GAFINet, the final diagnosis was confirmed as Tsutsugamushi disease in total 43 cases. The number of reported cases was small, and the epidemic peaked at the same time as the CDC surveillance data, but the outbreak occurred in the same period (October to November).

\section{Conclusions}

GAFINet has both the characteristics of indicator based surveillance and event based surveillance.

Data were collected over a period of one year to examine the feasibility and applicability of the indicator based surveillance. In comparison with national surveillance data, some feasibility was verified by similar trends, but the necessity of operating regional surveillance data still needs to be discussed. It should also be noted that some diseases have different peak times of one week.

The role of event based surveillance is mainly aimed at surveillance of fever patients with international travel history. The advantage of the GAFINet's classification system in the emergency room of participating hospitals is that the patient's cases of fever were constantly monitored. However, there is still a limit to the lack of budget and manpower in the samping and labaratory test.

Real time visual feedback of surveillance data helped to increase participation and discuss the results. 